\title{
Dienstplangestaltung aus ökonomischer Sicht des Pflegemanagements
}

\author{
Christa Meyer
}

(C) Springer-Verlag 2012

In den Krankenhäusern stehen heute mehr denn je ökonomische Aspekte im Mittelpunkt. Diese Anforderungen finden sich auch in der Personaleinsatzplanung (Dienstplan). Die pflegerische Versorgungskontinuität muss in der Patientenversorgung rund um die Uhr gewährleistet werden.

Für die Planung einer leistungsadaptierten Personalbesetzung (Schichtbesetzung) müssen geltende gesetzliche und tarifliche Vorgaben sowie klinik- und stationsinterne Anforderungen (fachlich, organisatorisch) berücksichtigt werden.

Besondere Anforderungen an die Personaleinsatzplanung haben Krankenhäuser der Maximalversorgung und Universitätskliniken durch den hohen Anteil der Notfallversorgung und das große Versorgungsspektrum im Rahmen ihres Versorgungsauftrages.

Insbesondere Universitätsklinken müssen sich der großen Herausforderung durch Forschung, Lehre und Krankenversorgung im Spannungsfeld zwischen Medizin und Ökonomie und dem Erhalt der Exzellenz in der wissenschaftlichen Weiterentwicklung, sowie der Gewährleistung einer professionellen Pflegequalität stellen.

$\mathrm{Zu}$ den Herausforderungen der Dienstplanung zählen einerseits die „harten“ Vorgaben, andererseits auch die Mitarbeiterzufriedenheit, z. B. unter dem Aspekt der Vereinbarkeit von Familie und Beruf sowie der Mitarbeiterorientierung im Rahmen einer lebensphasenorientierten Einsatzplanung. Daraus resultiert ein zunehmender Bedarf an Flexibilisierung der Dienstzeiten in Synchronisation mit dem leistungsadaptierten Arbeitsanfall auf der Station.

Unter dem Aspekt der patientenorientierten Prozessorientierung und bei gleichzeitiger ökonomischer Betrachtung ist

C. Meyer $(\bowtie)$

Vorstand für Krankenpflege und Patientenservice,

Universitätsklinikum Schleswig-Holstein, Kiel, Deutschland zukünftig auch der Einsatz unterschiedlicher Qualifikationen (Qualimix) im Stationsablauf gefordert.

Die Optimierung der kurz- und langfristigen Personaleinsatzplanung unter Berücksichtigung von ökonomischen, quantitativen und qualitativen Aspekten der Schichtbesetzung beeinflusst die Stabilität der Leistungsplanung in den Bereichen positiv und erhöht die Zufriedenheit von Patienten, Mitarbeitern und Arbeitgeber. Die Herausforderungen für eine effektive und effiziente Dienstplanung als Steuerungs- und Motivationsinstrument werden immer komplexer und sind ein relevantes Planungs- und Abrechnungsinstrument für Mitarbeiter und Führungskräfte. 